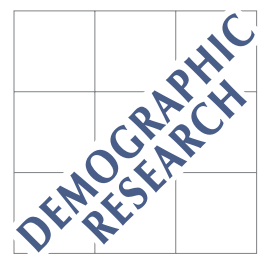

Demographic Research a free, expedited, online journal of peer-reviewed research and commentary in the population sciences published by the Max Planck Institute for Demographic Research Konrad-Zuse Str. 1, D-18057 Rostock · GERMANY www.demographic-research.org

DEMOGRAPHIC RESEARCH

VOLUME 20, ARTICLE 24, PAGES 595-598
PUBLISHED 29 MAY 2009

http://www.demographic-research.org/Volumes/Vol20/24/

DOI: $10.4054 /$ DemRes.2009.20.24

Formal Relationships 3

\title{
The effect of changes in fertility on the age distribution of stable populations
}

\section{Michel Guillot}

This article is part of the Special Collection "Formal Relationships".

Guest Editors are Joshua R. Goldstein and James W. Vaupel.

(C) 2009 Michel Guillot.

This open-access work is published under the terms of the Creative Commons Attribution NonCommercial License 2.0 Germany, which permits use, reproduction \& distribution in any medium for non-commercial purposes, provided the original author(s) and source are given credit. See http://creativecommons.org/licenses/by-nc/2.0/de/ 


\section{Table of Contents}

$1 \quad$ Relationship $\quad 595$

2 Proof $\quad 596$

$3 \quad$ Related relationship $\quad 596$

4 History $\quad 597$

5 Applications $\quad 597$

$\begin{array}{ll}\text { References } & 598\end{array}$ 


\title{
The effect of changes in fertility on the age distribution of stable populations
}

\author{
Michel Guillot ${ }^{1}$
}

\begin{abstract}
When fertility increases in a previously stable population, the new stable population will be younger than the old one, and the two age distributions will cross at the mean age of the populations.
\end{abstract}

\section{Relationship}

Consider a stable population with a growth rate $r$. Suppose that, in that population, agespecific fertility rates change to new levels and stay constant thereafter, while mortality remains constant. Eventually, a new stable population with a different growth rate will emerge. For example, if age-specific fertility rates increase, the growth rate of the new stable population will be higher than that of the old one. The effect of this fertility-induced change in $r$ on the proportionate age distribution of the stable population can be formally expressed as follows:

$$
\frac{d \ln [c(a)]}{d r}=A_{P}-a
$$

where $c(a) d a$ is the proportion of the stable population in the age interval $a$ to $a+d a$, and $A_{P}$ is the mean age of the stable population.

In Equation (1), the derivative of $\ln [c(a)]$ is positive at ages below $A_{P}$ and negative at ages above $A_{P}$. This means that, in the case of a fertility-induced increase in $r$, the proportion of the population at ages below $A_{P}$ will increase, while the proportion at ages above $A_{P}$ will decrease. At age $A_{P}$, the derivative is zero, so the proportion of the population at that age remains unchanged. In other words, the new stable population will be younger than the initial one, and the two age distributions will cross at the mean age of the populations. Conversely, in the case of a fertility-induced decrease in $r$, the new stable population will be older. Equation (1) also shows that the further away an age is from $A_{P}$, the larger the relative change in the age distribution.

\footnotetext{
${ }^{1}$ University of Wisconsin-Madison
} 


\section{Proof}

The age distribution of a stable population, $c(a)$, is given by

$$
c(a)=b e^{-r a} p(a)
$$

where $b$ is the crude birth rate, $r$ is the population growth rate, and $p(a)$ is the proportion surviving from birth to age $a$.

The impact of an infinitesimal, fertility-induced change in $r$ on $c(a)$ is analyzed by developing the derivative of $\ln [c(a)]$ with respect to $r$ :

$$
\frac{d \ln [c(a)]}{d r}=\frac{d[\ln b-r a+\ln p(a)]}{d r}
$$

Since $b=1 / \int_{0}^{\infty} e^{-r a} p(a) d a$ in a stable population, equation (3) becomes:

$$
\frac{d \ln [c(a)]}{d r}=\frac{d\left[\ln 1-\ln \int_{0}^{\infty} e^{-r a} p(a) d a-r a+\ln p(a)\right]}{d r} .
$$

This demonstration is about the impact on $c(a)$ of a fertility-induced change in $r$, so $p(a)$ is not affected and remains constant. Therefore, equation (4) simplifies as follows:

$$
\begin{aligned}
\frac{d \ln [c(a)]}{d r} & =\frac{-d \ln \int_{0}^{\infty} e^{-r a} p(a) d a}{d r}-\frac{d r a}{d r} \\
& =\frac{\int_{0}^{\infty} a e^{-r a} p(a) d a}{\int_{0}^{\infty} e^{-r a} p(a) d a}-a=\frac{\int_{0}^{\infty} a c(a) d a}{\int_{0}^{\infty} c(a) d a}-a \\
& =A_{P}-a
\end{aligned}
$$

Q.E.D.

\section{Related relationship}

Equation (1) pertains to an infinitesimal change in $r$ producing an infinitesimal change in $A_{P}$. When fertility changes are more substantial and produce non-infinitesimal changes in $r$, the old and new stable populations will have different mean ages. For these noninfinitesimal changes in $r$, the crossing age $\hat{A}$ is located between the mean ages of the old and new stable populations. For a pair of intrinsic growth rates that are in the range of $r$ values usually observed in human populations, the crossing age can be approximated by the mean of the mean ages of the two stable populations:

$$
\hat{A} \approx \frac{A_{P}^{1}+A_{P}^{2}}{2}
$$

where $A_{P}^{1}$ and $A_{P}^{2}$ are the mean ages of the old and new stable populations. 


\section{History}

Equation (1) and its proof first appear in Keyfitz (1968:186, equation (7.4.3)). A version of equation (1) is also discussed in Coale (1972:28, equation (2.27)). However, the foundations of Equation (1) can be traced back to Lotka (1938, 1939), who derived an expression for the crossing age, or "center of tilt", between the age distributions of two stable populations with identical mortality but different values of $r$ (Lotka 1939, equation (56)). He also showed that, for differences in $r$ in the range of .01, the crossing age can be well approximated using equation (6) (Lotka 1938:164-165), and demonstrated that, in the case of an instantaneous change in $r$, the crossing age is $A_{P}$ (1939, equation (58)). Later, Coale considered a wide range of life tables and estimated that, when the difference in $r$ between the two stable populations is less than .02, the exact value of the crossing age exceeds the approximation given in equation (6) by at most .16 years (Coale 1972:60, fn.7). Equation (1) is also discussed in Preston, Heuveline, and Guillot (2001:156-157).

\section{Applications}

Equation (1) elegantly shows that, in the long run, fertility increases produce younger populations, while fertility decreases produce older populations. By expressing the value of the crossing age, equation (1) also shows the ages at which changes in the age distribution are occurring. Lotka used his expression for the "center of tilt" to make the following practical point:

“(...) as we pass from greater to lesser values of $r$, the natural rate of increase, the proportion of the population comprised within the age groups at the young end and at the old end of the curve of the population change materially, but the age group or groups centered about the average age of the population, i.e., essentially the group of productive workers, undergoes relatively small changes." (Lotka 1938:166).

In reality, fertility changes occur in populations that are never perfectly stable. Moreover, mortality often varies in tandem with fertility. In such cases, equation (1) will not be applicable, and the effect of changes in fertility on the population age distribution needs to be studied with other approaches, for example using counterfactual projections or decomposition methods (Keyfitz 1968:189-193). Nonetheless, the overall message of equation (1), i.e., that fertility increases tend to make populations younger, holds in a variety of situations. 


\section{References}

Coale, A. (1972). The Growth and Structure of Human Populations: A Mathematical Investigation. Princeton NJ: Princeton University Press.

Keyfitz, N. (1968). Introduction to the Mathematics of Population. Reading, MA: Addison-Wesley Publishing Company.

Lotka, A. (1938). Some Recent Results in Population Analysis. Journal of the American Statistical Association 33(201): 164-178. doi:10.2307/2279123.

Lotka, A. (1939). Théorie Analytique des Associations Biologiques. Part II. Paris: Hermann et Cie.

Preston, S., Heuveline, P., and Guillot, M. (2001). Demography: Measuring and Modeling Population Processes. Oxford, UK: Blackwell Publishing. 